\title{
Trans-scale mechanics: looking for the missing links between continuum and micro/nanoscopic reality
}

\author{
Y. L. Bai · H. Y. Wang - M. F. Xia - F. J. Ke
}

Received: 21 January 2008 / Revised: 31 January 2008 / Accepted: 31 January 2008 / Published online: 13 March 2008 (C) Springer-Verlag 2008

\begin{abstract}
Problems involving coupled multiple space and time scales offer a real challenge for conventional frameworks of either particle or continuum mechanics. In this paper, four cases studies (shear band formation in bulk metallic glasses, spallation resulting from stress wave, interaction between a probe tip and sample, the simulation of nanoindentation with molecular statistical thermodynamics) are provided to illustrate the three levels of trans-scale problems (problems due to various physical mechanisms at macro-level, problems due to micro-structural evolution at macro/micro-level, problems due to the coupling of atoms/ molecules and a finite size body at micro/nano-level) and their formulations. Accordingly, non-equilibrium statistical mechanics, coupled trans-scale equations and simultaneous solutions, and trans-scale algorithms based on atomic/ molecular interaction are suggested as the three possible modes of trans-scale mechanics.
\end{abstract}

The project supported by the National Basic Research Program of China (2007CB814800), the National Natural Science Foundation of China (10432050, 10572139, 10721202, 10772012, 10772181, 90715001), and CAS Innovation Program (KJCX2-SW-L08, KJCX2-YW-M04).

Y. L. Bai · H. Y. Wang $(\bowtie) \cdot$ M. F. Xia · F. J. Ke

State Key Laboratory of Non-linear Mechanics (LNM),

Institute of Mechanics, Chinese Academy of Sciences,

Beijing 100080, China

e-mail: why@lnm.imech.ac.cn

\section{F. Xia}

Department of Physics, Peking University,

Beijing 100871, China

F. J. Ke

Department of Applied Physics, Beihang University,

Beijing 100083, China
Keywords Multi-scale $\cdot$ Missing-links $\cdot$ Continuum mechanics $\cdot$ Particle mechanics $\cdot$ Trans-scale mechanics

\section{Introduction}

Mechanics, consisting of particle and continuum mechanics, has played a significant role in the development of modern engineering. However, with incredibly extending engineering practices, for instance from static to high rate loadings, from macroscopic down to micro/nanoscopic technology and so on, engineers encounter more and more phenomena involving coupled multiple space and time scales, with which the conventional frameworks of either particle or continuum mechanics can hardly cope. Actually, the mechanics community owes engineers a lot for years on the unknown underlying mechanisms governing the phenomena with multiple scales. Take the problem of strength as an example, Tsien wrote in his well-known book Physical Mechanics [1], "there is another sort of problems, i.e., strength and plasticity theory, for which even essential physical formulation is still not available for engineering applications". Though some progresses have been made, till now physicists still share the same point of view: "despite the tremendous development of solid-state physics in this century, physicists have paid slight attention to how things break. In part, this neglect has occurred because the subject seems too hard. Cracks form at the atomic scale, extend to the macroscopic level, are irreversible, and travel far from equilibrium" [2]. In fact, we can name a lot of such phenomena in engineering, like how nanometerwide shear bands emerge from non-structured bulk metallic glasses (BMG's) [3]; why spallation resulting from stress waves does not follow either energy or impulse criteria macroscopically [4]; and how to efficiently simulate mechanical behaviors of materials under practical engineering 
loading rates (usually lower than $10^{6} / \mathrm{s}$ ) based on the entity of atoms (even in nano-technology) [5], etc. Obviously, all these practical problems offer a real challenge but also a wonderful opportunity for mechanics.

Now, let us have a close look at why trans-scale mechanics might become such a paradigm to cope with these tricky problems with coupled multiple space and time scales.

In ideal particle and continuum mechanics, there are object scales only. However, gravity and viscosity spoil geometrical similarity, implying a certain size effect. With brilliant foresight, Italian physicist Galileo (1564-1642) noticed that the combination of gravity and bone strength $\sigma_{c}$ constitutes an intrinsic length scale $\sigma_{c} / \rho g$, which sets an upper limit to an animal's size [6]. The real pioneering work with tremendous impact on modern industries (aviation) is Prandtl's theory on boundary layer [7]. To solve the d'Alembert's paradox of a body moving with no drag in fluid, Prandtl distinguished a thin layer with velocity gradient owing to viscosity on the moving body's surface from the main stream. The thickness of boundary layer can be evaluated by

$\delta \sim \sqrt{v t} \sim \sqrt{v \frac{X}{U}}$

where $v$ is kinetic viscosity, $U$ is the velocity of the main stream and $X$ is the distance in flow direction. Since the kinetic viscosity $v$ of air and water is in the range of $\left(10^{-6}\right.$ $\left.10^{-5}\right) \mathrm{m}^{2} / \mathrm{s}$, boundary layer will be $\mathrm{mm}$ thick, provided $U$ and $X$ are $\mathrm{cm} / \mathrm{s}$ and $\mathrm{cm}$, respectively. Noticeably, this is an emerging length scale, though still governed by macroscopic parameters of media, like kinetic viscosity. By coupling the thin boundary layer and the mainstream (much greater than the boundary layer), engineers successfully solved the problem of drag force with continuum mechanics and established modern aviation.

On the other hand, it is well known that solids are usually microscopically structured. The microstructure means the crystalline structure and all imperfections, including their size, shape, orientation, composition, spatial distribution, etc. As for the type of imperfections, it can include point defects (like vacancies and impurities), line defects (like edge and screw dislocations) and planar defects (like grain boundaries), second phase particles, etc. Among all these microscopical factors, the influence of grain size on mechanical properties draws special attention. The best known example may be the Hall-Petch relation, proposed in 1950s, between yield strength $\sigma_{Y}$ and the mean grain size $d$ [8],

$\sigma_{Y}=\sigma_{0}+\frac{k}{\sqrt{d}}$

where $\sigma_{0}$ and $k$ are two constants [8,9]. Till now, although 50 years has past, there is not a commonly accepted theory, which can satisfactorily explain the relation. Looking back

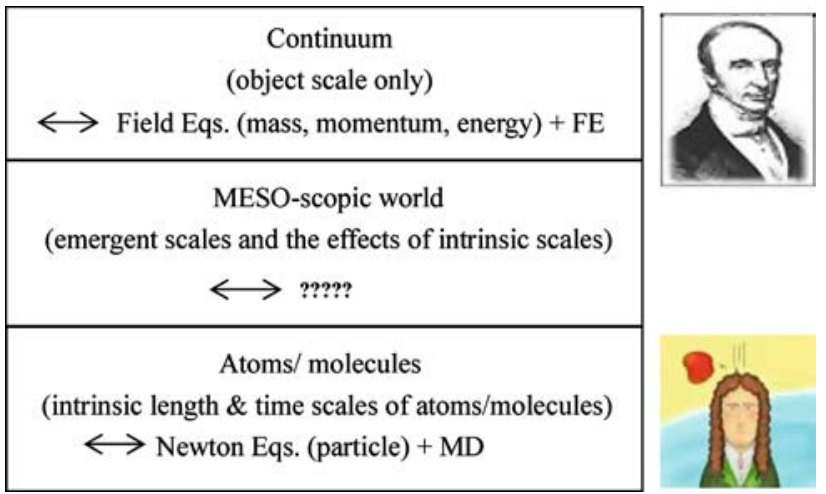

Fig. 1 An illustration of present mechanical formulations and the mesoscopic world

to the very few successful pioneering works and lots of remaining difficulties, we may have to ask ourselves if there are some links still missed in the present framework of mechanics. Perhaps, so far these missing links have been deeply rooted in the hierarchy of microstructures via intrinsic scales of length, time, energy, etc.

Figure 1 is a sketch illustrating how the present formulations of mechanics work and where the missing links might be. To understand the mechanisms governing mechanical properties, one can go down to atomic/molecular entity, for which Newton's equations of particles are in force and corresponding molecular dynamics (MD) algorithm works. When scaling up to continuum, the field equations together with constitutive relation are in force and corresponding finite element (FE) method works well. However, in between, namely on meso-scopical level, either conventional continuum mechanics or traditional Newton's equations of particles appears unable to properly cope with the rich emerging patterns and the critical roles of the intrinsic scales. Therefore, it seems that new formulations and corresponding numerical algorithms with trans-scale coupling are badly appealed for. To meet the need, a number of works on the problems appear recently worldwide, like multi-scale, trans-scale and mesomechanics $[10,11]$.

However, we have to confess that either multi-scale, transscale or mesomechanics is just a "provisional term" for the time being. As soon as a solid framework, which can properly unveil the missing trans-scale links, is established, it will have a more accurate name according to the nature of the missing links, like the appearance of thermodynamics, quantum mechanics, etc. So, the focus of trans-scale mechanics should be put on the exploration of the new physical essence of the missing links and relevant theoretical modes or paradigms.

In the following, we will use some case studies to illustrate three levels of trans-scale problems and their formulations. These are the problems: 
- due to various physical mechanisms (basically at macrolevel)—like the shear band formation in bulk metallic glasses (BMG);

- due to micro-structural evolution (basically at macro/ micro-level)—like the spallation resulting from stress wave;

- due to the coupling of atoms/molecules and a finite size body (basically at micro/nano-level)—-like the interaction between a probe tip and sample and the simulation of nanoindentation with molecular statistical thermodynamics.

Incidentally, in this paper the prefix nano- stands for the nanometer and atomic/molecular level, micro- for the micrometer and microstructural level and macro- for the level beyond millimeters, respectively. We wish, these case studies may highlight certain potential paradigms of trans-scale mechanics.

\section{Case studies}

Case 1: Shear band formation in bulk metallic glasses (BMG)

Metallic glasses with non-crystalline microstructures exhibit outstanding mechanical properties, such as high elastic limit, high fracture strength, etc. Recently, bulk metallic glasses (BMG's), centimeters or greater in size, have been successively synthesized. This presents many opportunities for engineering applications. However, the fundamental understanding of the deformation processes of BMG's is still lacking, for instance, the shear banding in BMG's.

In practice, localized shear bands are particularly important for metallic glasses, because they were observed under various loadings and may limit the application of bulk metallic glasses as structural materials. For a long time, there have been two types of mechanism proposed to explain the formation of shear bands in metallic glasses: the creation of free volume and the thermally assisted softening. Early in 1970s, Spaepen et al. indicated that shear-induced dilatation can reach dynamic equilibrium with no temperature rise [12, 13]. However, with their MD simulations Falk and Shi [14] demonstrated that "strain localization occurs in this system despite the lack of a measurable decrease in density", since "density fluctuations and small system size prevent us from directly detecting density changes smaller than approximately 3\%" [14]. Recently, Wang et al. [15] found in their MD simulations of metallic glass that the number density of atoms shifts to less condensed state under shear loading. Accordingly, shear instability owing to either free volume creation [16, 17] or coupled thermal softening and free volume creation was analyzed [18]. Experimentally, Bruck et al. [19] reported that "no adiabatic heating occurs before yielding" in

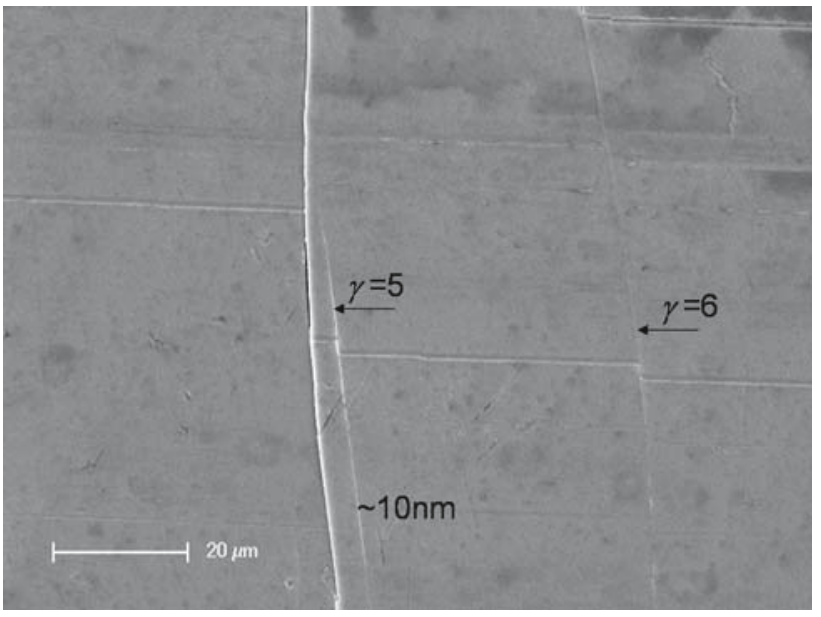

Fig. 2 SEM micrograph showing the width of shear band about ten $\mathrm{nm}$ in BMG's [25]

Zr-BMG; however, they observed that "temperature increases due to adiabatic heating occur after the onset inhomogeneous deformation." Recently Lewandowski and Greer [20] observed the temperature rise up to a $1000^{\circ}$. However, they indicated that "the temperature rise does not seem to control the shear band thickness". More significantly, TEM and SEM allow the measurements of shear band dimensions and the width of shear bands was typically about ten nm, as Fig. 2 shows [21-25]. But, till now the mechanism governing the emergence of nanometers wide shear bands in BMG's still remains open. Because of the nano meters feature in shear banding, this appeal for a trans-scale study of the phenomenon. So, the QUESTION for trans-scale study is: how nanometer-wide shear bands emerge in BMG's with noncrystalline microstructures?

The present trans-scale approach to the shear band formation in bulk metallic glasses is actually at macroscopic level, namely to translate the free volume creation at atomic level into a macroscopic version by introducing the free volume fraction $\xi$ and its creation and diffusion $[18,26]$

$\frac{\partial \xi}{\partial t}=D \frac{\partial^{2} \xi}{\partial y^{2}}+G(\xi, \theta, \tau)$

where $t$ is time, $y$ is the axis vertical to shearing, $D$ is the diffusivity of free volume and $G$ is the combined rate of free volume generation and annihilation, respectively. Then, we can combine the evolution of free volume fraction $\xi$, Eq. (3), with continuum mechanics equations (momentum and energy conservation),

$$
\begin{aligned}
& \rho \frac{\partial^{2} \gamma}{\partial t^{2}}=\frac{\partial^{2} \tau}{\partial y^{2}}, \\
& \frac{\partial \theta}{\partial t}=\kappa \frac{\partial^{2} \theta}{\partial y^{2}}+\frac{K \tau}{\rho C_{v}} \frac{\partial \gamma}{\partial t},
\end{aligned}
$$


Table 1 Typical parameters and variables of BMG's [26]

\begin{tabular}{|c|c|c|c|}
\hline $\begin{array}{l}\text { Parameters and } \\
\text { variables }\end{array}$ & Symbol & Value & Reference \\
\hline Density & $\rho$ & $5.9 \times 10^{3} \mathrm{~kg} \mathrm{~m}^{-3}$ & [27] \\
\hline Specific heat & $C_{p}$ & $532 \mathrm{~J} \mathrm{~kg}^{-1} \mathrm{~K}^{-1}$, at $\theta g$ & [27] \\
\hline Viscosity (kinetic) & $v$ & $\begin{array}{l}\sim 10^{-3} \mathrm{~m}^{2} \mathrm{~s}^{-1} \text { at } 1,000 \mathrm{~K} \\
\gg 10^{-3} \mathrm{~m}^{2} \mathrm{~s}^{-1}, \theta<\theta g\end{array}$ & {$[27,28]$} \\
\hline Thermal diffusivity & $\kappa$ & $3.5 \times 10^{-6} \mathrm{~m}^{2} \mathrm{~s}^{-1}$ & [27] \\
\hline $\begin{array}{c}\text { Free volume } \\
\text { diffusivity }\end{array}$ & $D$ & $<10^{-16} \mathrm{~m}^{2} \mathrm{~s}^{-1}$ & {$[28]$} \\
\hline Free volume creation & $G$ & $\sim\left(10^{-3}\right.$ to $\left.10^{-2}\right) \mathrm{s}^{-1}$ & [17] \\
\hline Free volume & $\xi$ & $\sim 10^{-2}$ & {$[17,29]$} \\
\hline Strain at yielding & $\gamma$ & $10^{-2}$ & {$[27,29]$} \\
\hline $\begin{array}{l}\text { Strain rate static } \\
\text { dynamic }\end{array}$ & $\dot{\gamma}$ & $\sim<10^{-3} \mathrm{~s}^{-1}$ to $10^{3} \mathrm{~s}^{-1}$ & {$[27,29]$} \\
\hline
\end{tabular}

and the constitutive relation

$\tau=\tau(\gamma, \dot{\gamma}, \theta, \xi)$

where $\rho$ is the density, $\gamma$ the shear strain, $\dot{\gamma}$ the shear strain rate, $\tau$ the shear stress, $\theta$ the temperature, $\kappa$ the thermal diffusivity, $C_{v}$ the specific heat, $K$ the Taylor-Quinney coefficient, respectively. Thus, the corresponding system of equations forms a complete framework governing the simple shear involving both thermal softening and diffusion as well as free volume creation and diffusion. Apart from the conventional continuum equations, the trans-scale consideration in the case is merely to take the free volume creation and diffusion, a phenomenon at atomic level, into account macroscopically.

The important points are that the parameters and variables involved in the trans-scale formulation imply several time and length scales [26]. The time scales are:

relaxation time $t_{R Q}=\frac{R}{Q}$,

external time $t_{e x}=\frac{\gamma}{\dot{\gamma}}$

compound free volume creation time scale $t_{G}=\frac{1}{G_{\xi}}$,

where $Q=(\partial \tau / \partial \gamma)$ is strain hardening, $R=(\partial \tau / \partial \dot{\gamma})$ is strain rate hardening and $G_{\xi}=(\partial G / \partial \xi)$ is compound creation of free volume, respectively. By definition, $t_{R} Q \sim$ $t_{e x}$ are in the order of $10^{1} \mathrm{~s}$ or $10^{-5} \mathrm{~s}$ under quasi-static or dynamic loadings, respectively. But, $t_{G}=\frac{1}{G_{\xi}}$ is different and closely related to the instability growth.

On the other hand, with a fixed time scale, for instance the relaxation time $t_{R Q}$, we can define three length scales, corresponding to 3 different diffusions (viscosity, heat and mass). They are $l_{\nu}^{2}=\frac{\nu R}{Q} \gg l_{\kappa}^{2}=\frac{\kappa R}{Q} \gg l_{D}^{2}=\frac{D R}{Q}$, since generally the kinetic viscosity $v>$ heat diffusion $\kappa>$ free volume diffusion $D$. Table 1 lists some typical values of relevant parameters and variables of BMG's. Keeping these scales in mind, now we turn to the analysis of shear banding.

To perform a linear stability analysis, we impose a small perturbation $\left(\tau^{\prime}, \gamma^{\prime}, \theta^{\prime}, \xi^{\prime}\right)$ on the smoothly developing homogeneous state $\left(\tau_{0}, \gamma_{0}, \theta_{0}, \xi_{0}\right)$, which is a solution of Eqs. (3)-(6), such that

$$
\begin{aligned}
& \{\tau, \gamma, \theta, \xi\}=\left\{\tau_{0}+\tau^{\prime}, \gamma_{0}+\gamma^{\prime}, \theta_{0}+\theta^{\prime}, \xi_{0}+\xi^{\prime}\right\}, \\
& \left\{\tau^{\prime}, \gamma^{\prime}, \theta^{\prime}, \xi^{\prime}\right\}=\left\{\tau^{*}, \gamma^{*}, \theta^{*}, \xi^{*}\right\} \mathrm{e}^{\alpha t+\mathrm{i} k y} .
\end{aligned}
$$

where $\left\{\tau^{*}, \gamma^{*}, \theta^{*}, \xi^{*}\right\}$ are the magnitudes of the perturbation, $\alpha$ is the rate of growth, and $k$ is the wave number, respectively. The stability of deformation is determined by the sign of the real part of $\alpha$. Substituting Eqs. (7) and (8) into Eqs. (3)-(6), we can obtain the characteristic equation for $\alpha$ [18],

$a_{4} \alpha^{4}+a_{3} \alpha^{3}+a_{2} \alpha^{2}+a_{1} \alpha+a_{0}=0$

with

$a_{4}=1$,

$a_{3}=\left[\beta+\rho C_{v}\left(D k^{2}-G_{\xi}\right)+\rho C_{v} G_{\tau} F\right] / \rho C_{v}$,

$a_{2}=\left[k^{2} \omega+\rho \beta\left(D k^{2}-G_{\xi}\right)+k^{2} \rho \lambda G_{\tau} F\right.$

$\left.+K \dot{\gamma} \rho G_{\theta} F\right] / \rho^{2} C_{v}$,

$a_{1}=k^{2}\left[\omega\left(D k^{2}-G_{\xi}\right)+\lambda Q k^{2}-K \tau G_{\theta} F\right] / \rho^{2} C_{v}$,

$a_{0}=k^{4} \lambda Q\left(D k^{2}-G_{\xi}\right) / \rho^{2} C_{v}$,

where $P=-(\partial \tau / \partial \theta)$ is thermal softening, $F=-(\partial \tau / \partial \xi)$, $G_{\theta}=(\partial G / \partial \theta), G_{\tau}=(\partial G / \partial \tau), \beta=K \dot{\gamma} P+\lambda k^{2}+R C_{v} k^{2}$ and $\omega=\lambda R k^{2}+\rho C_{v} Q-K \tau P$, respectively.

Since the complete instability analysis is quite complicated [26], here we just focus on the trans-scale effect of free volume creation and diffusion on shear banding. Firstly, the compound creation of free volume

$G_{\xi}=(\partial G / \partial \xi)>D k^{2}$

plays a key role in the occurrence of shear instability. Secondly, two simplified but informative expressions of quasisteady shear band width could be deduced from Eqs. (3) and (5) [26]

$$
\begin{aligned}
& \delta_{\text {free volume }} \sim \sqrt{D \frac{\xi^{*}}{G^{*}}}, \\
& \delta_{\text {Thermal }} \sim \sqrt{\kappa \frac{\gamma^{*}}{\dot{\gamma}^{*}}},
\end{aligned}
$$

where $*$ denotes the values within the shear band. From the data listed in Table $1, \delta_{\text {free volume }} \sim \mathrm{nm}$ and $\delta_{\text {Thermal }} \sim \mu \mathrm{m}$, respectively. After comparing to the boundary layer $\delta \sim$ $\sqrt{v t} \sim \sqrt{v \frac{X}{U}}$, one can notice that these three emergent bandlike structures are dominated by three different diffusions (mass, heat and viscosity) and corresponding time scales. 
Now, to conclude this case study, we would like to stress two points. The first is that nanometer-wide shear bands can emerge in BMG's owing to the combination of free volume creation and diffusion; the second is the trans-scale effect of the atomic events (free volume creation and diffusion) can be formulated macroscopically in association with conventional continuum mechanics.

Case 2: Damage evolution owing to microdamage nucleation and growth-coupled macro- and micro-scopic length and time scales

For most heterogeneous materials, such as alloys, ceramics, composites, rocks, etc. there are usually distributed microcracks or microvoids rather than a single macroscopic crack, as fracture mechanics has successfully dealt with. In this case, there are many length and time scales involved, relevant to the size of microdamage and its nucleation and growth rates.

Roughly speaking, microdamage, i.e., microcracks and microvoids, is formed at microscopic inhomogeneities. For example, in polycrystalline metals with $\mu \mathrm{m}$ grains, the microvoids or cracks of about $\mu \mathrm{m}$ usually nucleate in the grain boundaries or within the grains. The number density of such microdamage observed on the surface of metals may be in the range of $\left(10^{2}-10^{4} / \mathrm{mm}^{2}\right)$ [30]. Generally speaking, there are two basic and distinct processes of microdamage evolution: nucleation and growth, and each has its own distinctive mesoscopic kinetics. Since the observed macroscopic failure of metals is resulting from these processes with different rates, a trans-scale understanding of damage evolution becomes a necessity.

Spallation resulting from stress wave reflection is such an illustrative example of damage evolution with multiple space and time scales. From experimental observations, a universal criterion of time-integral for spallation has been used for a long time [31]

$$
\left(\frac{\sigma}{\sigma^{*}}-1\right)^{v} \cdot \Delta t=K,
$$

where $\sigma$ and $\sigma^{*}$ are stress and stress threshold, respectively, $\Delta t$ is the load duration, $v$ and $K$ are two parameters. This criterion indicates that the critical stress to spallation is no longer a material constant, but dependent on its loading duration. Furthermore, since the power exponent $v$ in the criterion is usually neither 1 nor 2 , the criterion is neither of momentum nor energy balance macroscopically [32]. Then what is the mechanism underlying the time-dependent failure.

Actually, this is a common difficulty in dealing with timedependent and multi-scale failure in solids. Meyers [33], Grady and Kips [34] stressed that "we still need quantitative/predictive models based on continuum measure of spalling and nucleation-and-growth of microcracks" [33] and "the continuum models based on the statistical nucleation and growth of brittle and ductile fracture appear to be an attractive approach" [34]. Above all, spallation is a typical process with coupled multiple space and time scales. At least, there are two length scales: the sample size at macroscopic level and the microdamage size at mesoscopic level. On the other hand, there are, at least, three time scales: the stress wave loading duration macroscopically, the two microscopic characteristic times: nucleation time and growth time of microdamage. So, the QUESTION for the trans-scale study is: what is the mechanism underlying spallation resulting from microdamage nucleation and growth?

Now, we outline our trans-scale approach to spallation, in terms of coupled macro- and microscopic formulation.

Based on the experimental measurements of microscopic kinetics of microdamage in spallation $[30,35,36]$, the two microscopic rates of microdamage nucleation $n_{N}$ and growth $\dot{c}$ are governed by its current and initial(nucleation) sizes $c$ and $c_{0}$ of microdamage as well as macroscopically local average stress $\sigma[30,36]$

$n_{N}=n_{N}\left(c_{0} ; \sigma\right)$,
$V=\dot{c}=V\left(c, c_{0} ; \sigma\right)$.

According to the two variables of microdamage: the current size $c$ and the nucleation size $c_{0}$, there are two number densities of microdamage: $n_{0}\left(t, c, c_{0}\right)$ is the number density of microdamage with current size $c$ and the nucleation size $c_{0}$ and $n(t, c)$ is the number density of all microdamage with current size $c$. The relation between the two number densities is,

$n(t, c)=\int_{0}^{c} n_{0}\left(t, c, c_{0}\right) \mathrm{d} c_{0}$.

The evolution equation governing the microdamage number density $n_{0}\left(t, c, c_{0}\right)$ is

$\frac{\partial n_{0}}{\partial t}+\frac{\partial\left(n_{0} \cdot V\right)}{\partial c}=n_{N}(c) \cdot \delta\left(c-c_{0}\right)$,

where $\delta\left(c-c_{0}\right)$ is Dirac $\delta$-function and has the same dimension as the reciprocal of microdamage size. The solution to the equation under constant stress $\sigma$ is [37]

$n_{0}\left(t, c, c_{0} ; \sigma\right)= \begin{cases}\frac{n_{N}\left(c_{0} ; \sigma\right)}{V\left(c, c_{0} ; \sigma\right)}, & 0<c<c_{f}(t), \\ 0, & \text { otherwise }\end{cases}$

where $c_{f}$ is the moving front of microdamage and defined by

$$
t=\int_{c_{0}}^{c_{f}(t)} \frac{\mathrm{d} c}{V\left(c, c_{0} ; \sigma\right)}
$$




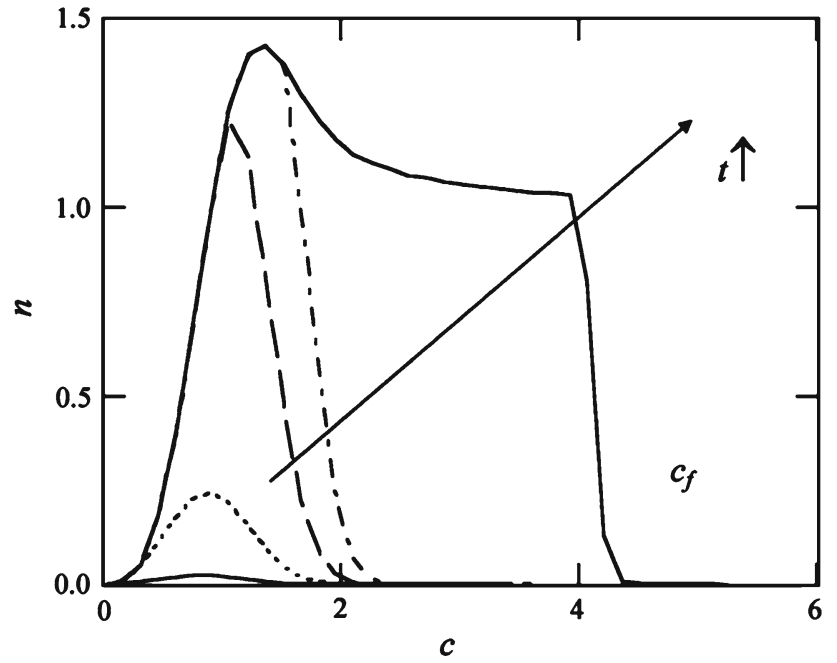

Fig. 3 The basic and unsteady solution of microdamage number density [37]

Therefore, the basic solution to the evolution of microdamage number density $n$ under constant stress $\sigma$ is [37]

$n(t, c ; \sigma)= \begin{cases}\int_{0}^{c} \frac{n_{N}\left(c_{0} ; \sigma\right)}{V\left(c, c_{0} ; \sigma\right)} \mathrm{d} c_{0}, & c<c_{f, 0}, \\ \int_{c_{0 f}}^{c} \frac{n_{N}\left(c_{0} ; \sigma\right)}{V\left(c, c_{0} ; \sigma\right)} \mathrm{d} c_{0}, & c>c_{f, 0},\end{cases}$

$t=\int_{c_{0 f}}^{c} \frac{\mathrm{d} c}{V\left(c, c_{0} ; \sigma\right)}$,

where $c_{f, 0}=c_{f}\left(t, c_{0}=0\right)$. This basic and unsteady solution of microdamage number density reveals two fundamental features of microdamage evolution: the saturation in the range of small microdamage size and an onward movement of microdamage front to larger size, see Fig. 3 [37].

Now, we turn to the unified set of equations combining continuum mechanics and microdamage evolution. For this sake, we should examine the evolution of microdamage number density in a continuum element at macroscopic coordinates $\boldsymbol{x}$. The coupled field equation of microdamage evolution is [38]

$\frac{\partial n}{\partial t}+\frac{\partial(n \cdot A)}{\partial c}+\frac{\partial(n \cdot \boldsymbol{v})}{\partial \boldsymbol{x}}=n_{N}$,

where $A$ is the average growth rate of microdamge with current size $c$,

$A(t, c ; \sigma)=\frac{\int_{0}^{c} V\left(c, c_{0} ; \sigma\right) \cdot n_{0} \mathrm{~d} c_{0}}{n(t, c ; \sigma)}$.

Note that the two spaces, namely the microdamage space $c$ and continuum space $\boldsymbol{x}$ are coupled with each other in the unified formulation

However, for engineering applications, what one would like to see is the effect of microdamage on macroscopic behavior of materials, rather than the microdamage itself.
So, we should convert the number density of microdamage into continuum damage $D$, for instance by

$D(t, \boldsymbol{x})=\int_{0}^{\infty} n(t, \boldsymbol{x}, c) \cdot \tau \cdot \mathrm{d} c$,

where $\tau$ is the failure volume of an individual microdamage with size $c$.

Then, the above unified set of equations combining continuum mechanics and microdamage evolution can be transformed into a form suitable to engineering applications, but still containing enough information of microdamage evolution. The one dimensional version of such an associated system of equations is (for simplicity, energy equation is omitted for the time being)

$$
\begin{aligned}
& \frac{\partial D}{\partial T}+D \frac{\rho}{\rho_{0}} \frac{\partial v}{\partial Y}=f, \\
& \frac{\partial \rho}{\partial T}+\frac{\rho^{2}}{\rho_{0}} \frac{\partial v}{\partial Y}=0, \\
& \frac{\partial v}{\partial T}-\rho_{0}^{-1} \frac{\partial \sigma}{\partial Y}=0, \\
& \sigma_{s}=\sigma_{s}(\varepsilon), \\
& \sigma=\frac{\sigma_{s}(\varepsilon)}{1-D}
\end{aligned}
$$

where $f$ is the dynamic function of damage (DFD),

$$
\begin{aligned}
f= & \int_{0}^{\infty} n_{N}(c ; \sigma) \cdot \tau \cdot \mathrm{d} c \\
& +\int_{0}^{\infty} n(t, Y, c ; \sigma) \cdot A(t, c ; \sigma) \cdot \tau^{\prime} \cdot \mathrm{d} c,
\end{aligned}
$$

which includes all necessary information of the effects of microdamage nucleation and growth on continuum damage evolution, $\tau^{\prime}=\mathrm{d} \tau / \mathrm{d} c$ and $\sigma_{s}$ is true stress.

Obviously, the DFD function $f$ is a convenient and informative agent bridging microscopic kinetics of microdamage and continuum damage. Additionally, the field equation of damage evolution (30) is different from the conventional continuum damage mechanics, in which there is not any microscopic kinetics of microdamage introduced explicitly.

Now let us take a close examination on the mechanisms underlying the trans-scale coupling in damage evolution. In most practical cases, the microstructural length scale, like the typical microdamge size $c^{*}$, is much less than the macroscopic sample size $L$,

$R=\frac{c^{*}}{L} \ll 1$.

Now, why such a small microstructural length scale can affect macroscopic behaviors? 
As a matter of fact, there are several competing macroscopic and microscopic time scales and they form the coupled trans-scale effects of multiple length and time scales. When the governing equations are rewritten in dimensionless form, two trans-scale dimensionless numbers appear, one can be called as the imposed Deborah number $D e^{*}=\frac{a c^{*}}{L V^{*}}$ and the other is the intrinsic Deborah number $D^{*}=\frac{n_{N}^{*} c^{* 5}}{V^{*}}$, where $a$ is the acoustic speed, $c^{*}, V^{*}$ and $n_{N}^{*}$ are characteristic microdamage size, growth rate and nucleation rate, respectively $[39,40]$.

The imposed Deborah number $D e^{*}$ appears preceding the DFD function in the dimensionless damage evolution equation and hence characterizes the continuum damage evolution rate,

$\frac{\partial \bar{D}}{\partial \bar{T}}+\frac{\bar{D}}{1+\varepsilon} \frac{\partial \bar{v}}{\partial \bar{X}} \approx D e^{*-1} \bar{f}$.

Actually, $D e^{*}$ is the ratio between the characteristic growth time of microdamage $t_{V}^{*}=\frac{c^{*}}{V^{*}}$ and the stress wave duration in the sample $t_{i}=\frac{L}{a}$, namely $D e^{*}=\frac{a c^{*}}{L V^{*}}=\frac{t_{V}}{t_{i}}$. In particular, the ratio of length scales at micro-and macro-levels $R=c^{*} / L$ does not independently appear in the governing equations but is included in the imposed Deborah number $D e^{*}$ as a combination of two ratios: the size scale ratio $R=c^{*} / L$ and the ratio of two velocities $V^{*} / a$. Hence, the imposed Deborah number $D e^{*}$ represents the trans-scale competition between the macroscopically imposed wave loading and the intrinsic microdamage growth. In the case of spallation, $D e^{*}<1$ and this means that microdamage has enough time to grow during the macroscopic wave loading and the microdamage growth predominates spallation.

On the other hand, the intrinsic Deborah number $D^{*}$ is the ratio between the characteristic growth time $t_{V}^{*}=\frac{c^{*}}{V^{*}}$ and the characteristic nucleation time of microdamage $t_{N}=\frac{1}{n_{N}^{*} c^{* 4}}$, namely $D^{*}=\frac{t_{V}}{t_{N}}=\frac{n_{N}^{*} c^{* 5}}{V^{*}}$. Provided damage localization can be formulated by the condition [37],

$\frac{\partial f}{\partial D} \geq \frac{f}{D}$.

One can find that the intrinsic Deborah number $D^{*}$ appears preceding the critical damage for damage localization and represents the critical damage, Fig. 4.

$$
\begin{aligned}
& D_{\text {localization }} \\
& =D^{*} \cdot \frac{\int_{0}^{\infty} \bar{\tau}\left(\bar{c}_{f}\right) \bar{n}_{N}\left(\bar{c}_{0}\right) \mathrm{d} \bar{c}_{0} \cdot \int_{0}^{\infty} \frac{\bar{\tau}\left(\bar{c}_{f}\right) \bar{n}_{N}\left(\bar{c}_{0}\right)}{V\left(\bar{c}_{0}, \bar{c}_{f}\right)} \mathrm{d} \bar{c}_{0}}{\int_{0}^{\infty} \bar{\tau}^{\prime}\left(\bar{c}_{f}\right) \bar{n}_{N}\left(\bar{c}_{0}\right) \mathrm{d} \bar{c}_{0}} \\
& =D^{*} \cdot O(1) .
\end{aligned}
$$

In fact, it is found in simulations that the critical damage to localization in spallation is about $\left(10^{-3}-10^{-2}\right)$, i.e., in the order of $D^{*}[37,41]$. Finally, this small $D^{*}$ indicates that the

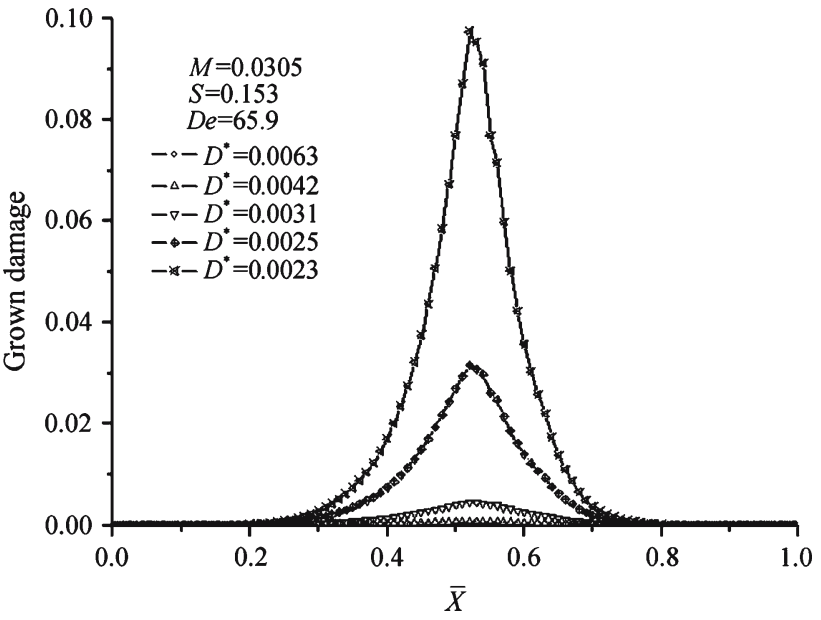

Fig. 4 The intrinsic Deborah number $D^{*}$ represents the critical damage to localization [42]

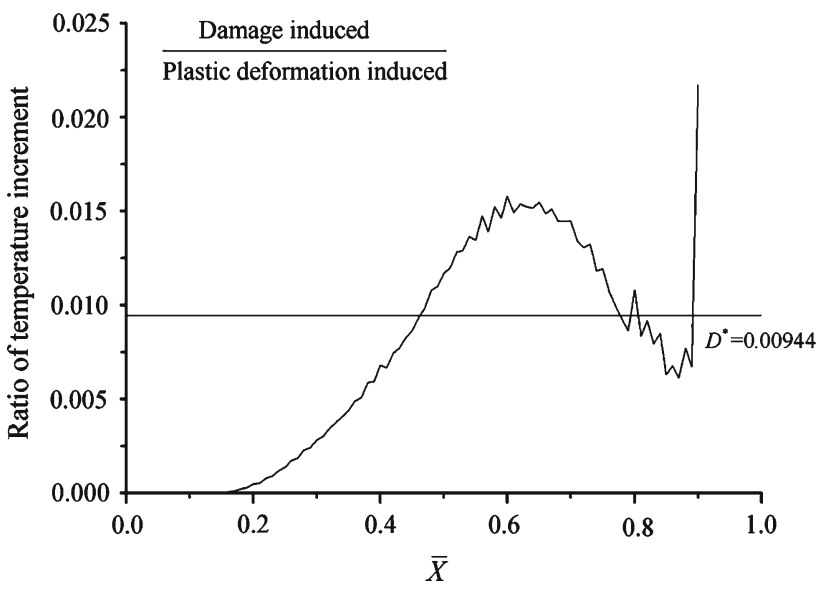

Fig. 5 The intrinsic Deborah number $D^{*}$ represents the energy dissipation due to microdamage compared to the bulk plastic work [42]

energy dissipation due to microdamage is negligible compared to the bulk plastic work. This explains why spallation can not be formulated by macroscopic energy criterion but must be treated with trans-scale analysis, Fig. $5[40,42]$.

In one word, in the case study of spallation, there are three physical processes with three time scales at two spatial levels (sample size $L$ and microdamage size $c^{*}$ ): the macroscopic imposed time scale $t_{i}=L / a \sim 10^{-6} \mathrm{~s}$ and two microscopic time scales, growth time scale $t_{V}=c^{*} / V^{*} \sim 10^{-6} \mathrm{~s}$ and the nucleation time scale $t_{N}=\left(n_{N}^{*} c^{* 4}\right)^{-1} \sim 10^{-3}$ s, see Table 2 . They can form two independent Deborah numbers governing the trans-scale coupling. This implies that the competition of the three macro- and microscopic rates is the trans-scale mechanism underlying spallation.

Case 3: Interaction between a probe tip and liquid surfacemechanics involving atomic/molecular interaction

The probe sensing technique, like atomic force microscope (AFM), nanoindentation, etc., has been widely used in 
Table 2 Parameters and time scales involved in spallation [42]

\begin{tabular}{lllll}
\hline & Quantity & Symbol & Dimension & Magnitude \\
\hline Macroscopic parameters & Sample size & $L$ & $\mathrm{~L}$ & $10^{-2} \mathrm{~m}$ \\
& Density & $\rho$ & $\mathrm{ML}^{-3}$ & $10^{3} \mathrm{~kg} / \mathrm{m}^{3}$ \\
& Elastic wave speed & $a$ & $\mathrm{LT}^{-1}$ & $10^{3} \mathrm{~m} / \mathrm{s}$ \\
& Characteristic stress & $\sigma_{Y}$ & $\mathrm{ML}^{-1} \mathrm{~T}^{-2}$ & $10^{8} \mathrm{~Pa}$ \\
& Impact velocity & $v_{f}$ & $\mathrm{LT}^{-1}$ & $10^{2} \mathrm{~m} / \mathrm{s}$ \\
Microdamage parameters & Characteristic size & $c^{*}$ & $\mathrm{~L}^{-6} \mathrm{~m}$ \\
& Growth rate & $V^{*}$ & $\mathrm{LT}^{-1}$ & $10^{0} \mathrm{~m} / \mathrm{s}$ \\
& Nucleation rate & $n_{N}^{*}$ & $\mathrm{~L}^{-4} \mathrm{~T}^{-1}$ & $10^{4} \mathrm{~mm}{ }^{-3} \mu \mathrm{m}^{-1} \mu \mathrm{s}^{-1} \mathrm{to} 10^{25} \mathrm{~m}^{-4} \mathrm{~s}-1$ \\
& Wave duration & $t_{i}$ & $\mathrm{~T}^{-1}$ & $10^{-6} \mathrm{~s}$ \\
& Growth time & $t_{V}$ & $\mathrm{~T}^{-1}$ & $10^{-6} \mathrm{~s}$ \\
& Nucleation time & $t_{N}$ & $\mathrm{~T}^{-1}$ & $10^{-3} \mathrm{~s}$ \\
\hline
\end{tabular}

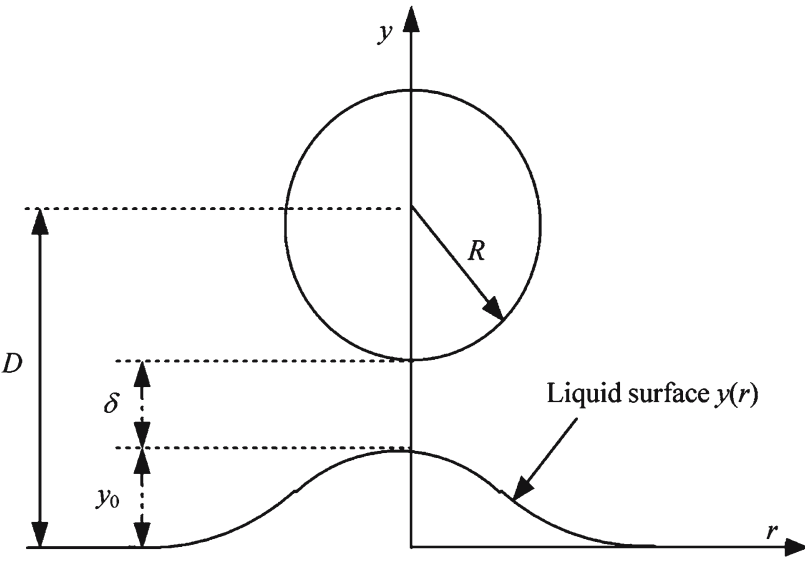

Fig. 6 A schematic illustration of the deformation of a liquid surface [44]

various fields. However, in practice, liquid film may appear on the surface of sample. Owing to the flowability of liquid, this film will no longer remain flat under its interaction with probe and this will inversely affect the interaction between the probe tip and the liquid, then the measured image of the sample will be distorted [43]. In particular, at a critical gap between the tip and the liquid dome, $\delta_{c}$, the liquid will jump up to connect to the tip and form a liquid bridge. Obviously, this critical jump is governed by a trans-scale combination of the tip size, gravity, surface tension and the atomic/molecular interaction (mainly van der Waals force) between the tip and liquid. So, the QUESTION for the trans-scale study is: what is the mechanism underlying the shape and critical jump of liquid surface under a probe tip?

Now, we turn to a simple but enlightening example: the interaction between a sphere tip and a semi-infinite liquid, owing to the atomic/molecular interaction. As shown in Fig. 6, the shape of the liquid dome $y(r)$ under a sphere tip with radius $R$ can be formulated by the equation [44],

$$
\begin{aligned}
\gamma_{L V} & \frac{\mathrm{d}}{\mathrm{d} r}\left[r \frac{y^{\prime}}{\left(1+y^{\prime 2}\right)^{1 / 2}}\right] \\
& +\frac{4 A R^{3}}{3 \pi} \frac{r}{\left[(D-y)^{2}+r^{2}-R^{2}\right]^{3}}-\rho g r y=0,
\end{aligned}
$$

where $A \sim 10^{-19} \mathrm{~J}$ is Hamaker constant [45], $\gamma_{L V}$ is the surface tension between liquid and air, $\rho$ is density, $g$ is gravity, respectively. There are three forces included: surface tension, gravity and intermolecular force (via Hamaker constant $A$ ). More significantly, these forces imply three very distinct length scales, namely 3 orders between each two adjacent length scales, see Table 3.

This trans-scale equation can be solved by employing the matching method proposed by Prandtl. The shape of liquid surface far from the probe is [44],

$y(r)=-C K_{0}\left(\frac{r}{\lambda}\right)$,

where $K_{0}$ is the modified Bessel function of the second class and $C$ is a constant. Clearly, the capillary length $\lambda=$ $\sqrt{\frac{\gamma_{L V}}{\rho g}} \sim$ mm characterizes the horizontal scale of the liquid dome. However, the central height of the liquid surface is,

$y_{0}=\frac{A R^{3}}{3 \pi \gamma_{L V}} \frac{\left(\frac{1}{2}+\ln \frac{2 \lambda}{\sqrt{\left(D-y_{0}\right)^{2}-R^{2}}}-E u\right)}{\left[\left(D-y_{0}\right)^{2}-R^{2}\right]^{2}}$,

where $E u \approx 0.57721$ is Euler's constant. Obviously, the height of the dome $y_{0}$ is dominated by the combination of van der Waals force (via Hamaker constant $A$ ) and surface tension $\gamma$ as well as the tip radius $R$, but nearly no business with gravity. In particular, the critical dome height for the surface jump up to contact the tip is

$y_{0 c} \sim \delta_{c} \sim\left(R \cdot \frac{A}{\gamma_{L V}}\right)^{1 / 3}$. 
Table 3 Three length scales involved in the interaction between a probe tip and liquid surface

\begin{tabular}{llll}
\hline & Symbol and formula & Magnitude & Essence \\
\hline Capillary length & $\lambda=\sqrt{\frac{\gamma_{L V}}{\rho g}}$ & $\sim \mathrm{mm}$ & $\begin{array}{c}\text { The combination of gravity } \\
\text { and surface tension }\end{array}$ \\
Tip radius & $R$ & $\sim \mu \mathrm{m}$ & $\begin{array}{l}\text { The combination of van der Waals force } \\
\text { Nanoscopic }\end{array}$ \\
$\begin{array}{lll}\text { length scale } \\
l_{A \gamma}=\sqrt{\frac{A}{\gamma_{L V}}}\end{array}$ & $\sim \mathrm{nm}$ & (via Hamaker constant) and surface tension \\
\hline
\end{tabular}

The partition of the two length scales $R$ and $l_{A \gamma}$ in the critical height is neither arithmetic nor geometric average, but in a way very similar to the well-known Tabor number $\mu$ in solid contact:

$\mu=\left(R \cdot \frac{\varpi^{2}}{E^{* 2} \xi_{0}^{3}}\right)^{1 / 3}=\left(R \cdot \frac{\varpi^{2}}{E^{* 2}}\right)^{1 / 3} / \xi_{0}$,

where $E^{*}$ is the reduced modulus, $\varpi$ is the work of adhesion, $\xi_{0}$ is interatomic equilibrium distance at tip-sample interface, respectively. So, this partition may be quite universal in this sort of trans-scale problems.

Now, we turn to a general intermolecular potential $w$ of two atoms with distance $\eta$

$w(\eta)=-C / \eta^{n}$

where $n$ is the power-law index and governs the attractive interaction between molecules. The fascinating trans-scale aspect is that the ratio of the tip-dome gap $\delta_{c}$ and dome height $y_{0 c}$ at critical jump, namely $\delta_{c} / y_{0 c}$ is closely related to the power index $n$,

$\frac{\delta_{c}}{y_{0 c}} \approx(n-4)$, when $\delta_{c} \ll R$.

Clearly, for van der Waals interaction $n=6$, the critical gapheight ratio is to 2 , as previously obtained [46]. This means that the intermolecular power index $n$ persists its trans-scale presentation under a micrometer tip $[47,48]$.

To summary the trans-scale case study of the tip-liquid interaction, two aspects are worth noticing. The first is that the liquid dome height is dominated by two length scales, the nanoscopic length scale and the tip radius as $y_{0} \sim \delta \sim$ $\left(R \cdot \frac{A}{\gamma_{L V}}\right)^{1 / 3}$, but is neither their arithmetic nor geometric average. The second is that the power index of intermolecular interaction $n$ presents itself very robust in the ratio between the dome height and tip-dome gap at critical jump under a micrometer tip. This trans-scale appearance of intermolecular interaction at a higher length level is worth further thinking.

Case 4: Simulation of quasi-static deformation at finite temperature based on atomic/molecular interaction-the variation of nanohardness with indentation depth
Table 4 Comparison of length and time scales in micro/nano engineering and molecular/atomic interaction

\begin{tabular}{lll}
\hline & Length scale & Time scale \\
\hline Micro/nano engineering & $10^{-6} \mathrm{~m}$ to $10^{-9} \mathrm{~m}$ & Duration: $\mathrm{s}-$ min \\
Molecular interaction & $10^{-10} \mathrm{~m}$ & Period: $t^{*} \sim 10^{-13} \mathrm{~s}$ \\
\hline
\end{tabular}

Now, molecular dynamics (MD) are the orthodox means for simulating molecular-scale models of matter [49]. Its essence is very simple: successive configurations of the atomic system are generated by integrating Newton's laws of motion of all molecules in a concerned system, based on molecular potential, like L-J potential,

$w=4 \varepsilon_{0}\left[\left(\frac{r_{0}}{r}\right)^{12}-\left(\frac{r_{0}}{r}\right)^{6}\right]$

where $r_{0} \sim 10^{-10} \mathrm{~m}$ is the range of interaction and $\varepsilon_{0} \sim$ $10^{-19} \mathrm{~J}$ is the characteristic energy However, this essence also sets a limit on MD applications. Since the integration of Newton's laws is of dynamics, the time step in the integration must be less than the oscillatory period of molecules $t^{*}$, namely $\Delta t \ll t^{*} \sim \sqrt{\left(\varepsilon_{0} / m r_{0}^{2}\right)} \sim 10^{-13}$ s, where $m$ is the molecule mass. For most engineering problems, like nanoindentation, the process usually last over minutes, hence appears to be quasi-static. So, there is a huge gap in time scales between MD simulation and engineering practices. On the other hand, even in nano-indentation, one still have to deal with the huge amount of molecule in MD simulations, for instance a micrometer cube would have $10^{12}$ atoms in metals, see Table 4. Obviously, this huge gap in temporal and spatial scales between practices and simulations offers MD a real challenge, let alone the unaffordable time consumption in large scale MD computations. In addition, MD simulations are sensitively dependent on the unknown initial conditions of atoms.

To illustrate the significance of the trans-scale analysis, let us have a close look at nanoindentation.

Nanoindentation is a powerful tool in the investigation of nano- and micro-scale mechanical properties of materials and has been widely used in biology, materials and mechanical engineering [50]. In recent years, nano-indentation tests have 
Fig. 7 Relation of nanohardness vs. indentation depth. a Experimental [54]; b MD simulation [55]
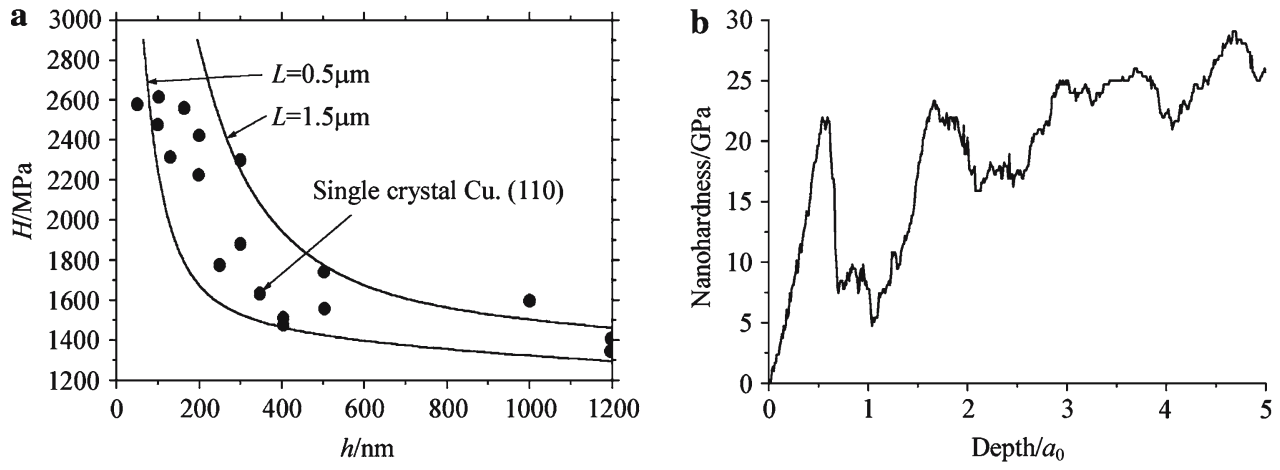

shown that the hardness at sub-micrometer scale is no longer a conventional constant but increases significantly with decreasing indentation depth [51]. Take single crystal copper as an example. A nearly doubled increase in nano-hardness from micrometer down to a few 100 nanometers of indentation depth has been reported [52-54]. One would like to ask what would be the limit of nano-hardness when the indentation depth tends to zero. Unfortunately, since both load $F$ and contact area $A$ tend to zero when the indentation depth $h$ approaches zero, the hardness can become any value by definition, as shown below,

$\lim _{h \rightarrow 0} H(h)=\frac{F(h)}{A(h)}=\frac{0}{0}$.

In order to solve the tricky problem, some researchers turn to MD simulations of nano-indentation to look for the answer (for instance [55,56]). They found that the hardness starts from zero at zero indentation depth, increases to a peak value and then seems to increase with further increasing indentation depth in a nanometer range, as shown in Fig. 7 [55].

So, when putting the experimental results and the MD simulations together, one may anticipate that the variation of nanohardness would be as follows: the hardness at micrometer depth remains a constant, then increases with decreasing indentation depth and reaches a peak value somewhere, finally decreases to zero when the indentation depth tends to zero. However, the experimental and numerical results forming the above picture of hardness variation have too huge differences in time and length scales: the experimental one was performed in 100 nanometers indentation depth and quasi-statically, whereas MD simulations were limited to nanometer and sub-nanosecond. These huge temporal and spatial differences make the above anticipation of nanohardness variation not so convincing. As a matter of fact, this appeals for a unified trans-scale mechanics and algorithm to bridge the huge gap of length and time scales from atomic scales to practical measurement. So, the QUESTION for the trans-scale study is: how to formulate a unified algorithm, which can reveal the full variation of nano-hardness under quasi-static loading at room temperature based on atomic interaction?
Recently, Dupuy et al. [57] and Hu and Wang et al. [58,59], briefly reported their quasi-static approaches to molecular simulation at finite temperature. The approach of FiniteTemperature Quasicontinuum [57], is based on their previous quasicontinuum (QC) method with the concept of repatoms, a zero-temperature minimization technique. The approaches proposed by Hu and Wang et al. [58-60], the molecular statistical thermodynamics (MST)/cluster statistical thermodynamics (CST), is based on the statistical thermodynamics formulation of Helmholtz free energy of atoms and its minimization.

As Born and Huang [61] pointed out that in many aspects the behavior of an atomic system are identical to a system of oscillators, for which the total of oscillations should be equal to 3 times of the total atoms, i.e., $3 N$. That is to say, the atoms could be treated as particles at their equilibrium positions when the mechanical deformation of the atomic lattice is examined, while the atoms should be treated as oscillators with various frequencies when the contribution of the thermal oscillations of atoms to deformation is examined. The particle-oscillator duality of atoms is the basis of MST method [58-60]. In accord with the concept of oscillators, statistical thermodynamics gives the Helmholtz free energy $A$ of $N$ atoms as the function of oscillatory frequency $\omega_{i \xi}$ [61],

$A=\Phi+k T \sum_{i=1}^{N} \sum_{\xi=1}^{3} \ln \left[2 \sinh \left(\frac{1}{2} \frac{\hbar \omega_{i \xi}}{k T}\right)\right]$,

where $\Phi$ is the static lattice energy, $T$ is temperature, $\hbar$ is Planck's constant, $k$ is Boltzmann's constant, $\omega_{i \xi}(\xi=$ $1,2,3)$ are the three oscillating frequencies of atom $i$, respectively.

Now, the key to the application of the Helmholtz free energy $A$ to the simulation of deformation becomes how to properly formulate the oscillating frequencies. Actually, there have been some well-known theories on the issue.

Einstein assumed that all oscillators have the same frequency, however, this can represent the isotropic oscillation of independent atoms only. After considering the constraint of atoms in lattice, Debye proposed that the $3 N$ frequencies 
Fig. 8 Schematic diagram of the MST/CST set-up for nanoindentation simulation [60]. a MST/CST simulation of nanoindentation; b Atomic configuration of the cone indenter in MST/CST simulation

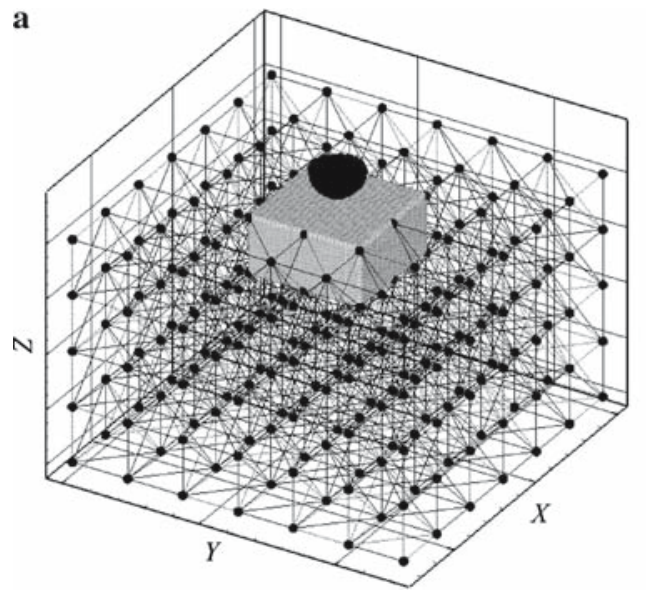

b

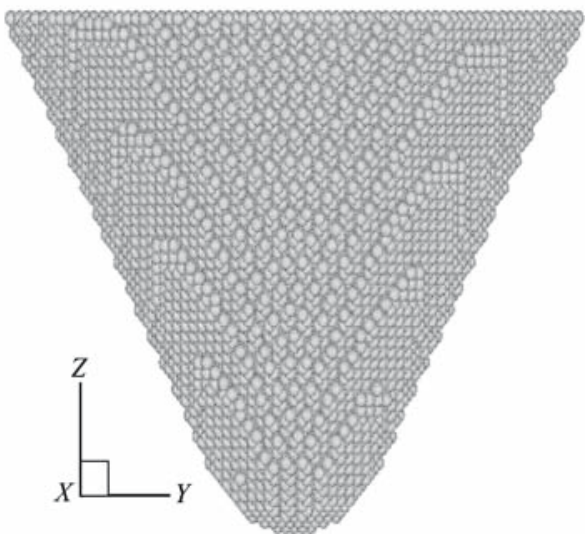

could be taken as the $3 \mathrm{~N}$ lowest frequencies of a continuum, which possesses the same elastic constants as the atomic lattice. Debye's theory has had great success in the explanation of thermal properties of solids. However, it is found that neither Debye nor Einstein approximation is able to reflect severely localized deformation. As noted by Tsien [1] that Debye's theory is still not an accurate approximation, and once there are differences between Debye's approximation and facts, one should resort to other formulations. As mentioned above, the atomic oscillators have very high frequencies and hence very short time scales, like $10^{-13} \mathrm{~s}$. So, the key to MST simulation of quasi-static deformation is to find a proper agent to take the place of the high frequencies in the expression of Helmholtz free energy.

According to lattice dynamics [61], the oscillating frequencies $\omega_{i \xi}$ can be taken as the $3 N$ eigenvalues obtained from the diagonalization of the dynamical matrix $D$,

$D_{i j}^{\xi \eta}=\frac{1}{\sqrt{m_{i} m_{j}}} \frac{\partial^{2} \Phi}{\partial x_{i \xi} \partial x_{j \eta}}$,

where $m_{i}$ is the mass of atom $i, x_{i \xi}$ is the $\xi$ th coordinate of atom $i$. Hence, the coupling of the two representations of atoms, i.e., particles and oscillators, makes it possible to determine the equilibrium positions of atoms at finite temperature by minimizing Helmholtz free energy with respect to the coordinates of all atoms,

$\delta A\left(\boldsymbol{x}_{i}\right)=0$.

As a matter of fact, in MST simulations of quasi-static deformation at finite temperature, the equilibrium configuration of the atoms is determined by minimizing the Helmholtz free energy under the condition of constant number of atoms $(N)$, constant temperature $(T)$ and constant volume $(V)$. Thus, for successive increments of boundary displacement, the successive corresponding equilibrium configurations of the atoms can be obtained similarly, therefore, and form a series of quasi-static deformation patterns. In this way, the gap between quasi-static process and high oscillatory frequencies of atoms is solved. The simulations of some typical 2D and 3D deformations showed that MST method needs one tenth of computation time used in MD simulations only. The trick of the high efficiency in MST is due to the explicit formulation of thermal contribution to Helmholtz free energy. In addition, at classical assumption $(h v \ll k T)$ the thermal energy is just the function of temperature, and the dependence on the atomic configuration appears in potential energy and entropy only. This is what the real essence of mechanical deformation is.

In order to bridge the gap between the size of practical test-piece (usually greater than nanometers) and the atomic length scale $\left(10^{-1} \mathrm{~nm}\right)$, coupled molecular/cluster statistical thermodynamics (MST/CST) method was introduced [59]. One attractive feature of this method is its "seamlessness", in which the same underlying atomistic potential is adopted in all regions, either individual atoms or clusters of atoms. On the other hand, compared to MD simulations, their high computational efficiency appears to be very promising.

Since the MST/CST method can bridge the huge gap of both temporal and spatial scales, from $10^{-13} \mathrm{~s}$ and $10^{-10} \mathrm{~m}$ of atomic interaction to the quasi-static process of a system tens nanometers large, we apply the method to the quasistatic nano-indentation test at room temperature, in order to provide a panoramic picture of the variation of nano-hardness with indentation depth.

In the simulation, a single crystal $\mathrm{Cu}$ test piece is indented by a cone diamond indenter at $300 \mathrm{~K}$ (Fig. 8). The cone indenter has a $60^{\circ}$ cone angle and a spherical tip with radius $2 \mathrm{~nm}$, hence the indenter is actually a truncated cone with a 1 $\mathrm{nm}$ high spherical crown. The dimension of the single crystal $\mathrm{Cu}$ test piece is $37.6 \mathrm{~nm} \times 37.6 \mathrm{~nm} \times 18.8 \mathrm{~nm}$, containing $2,293,253$ atoms, and its top surface is the (001) plane and the other two side surfaces are (100) and (010) planes, respectively. The top surface is traction free, and the bottom 


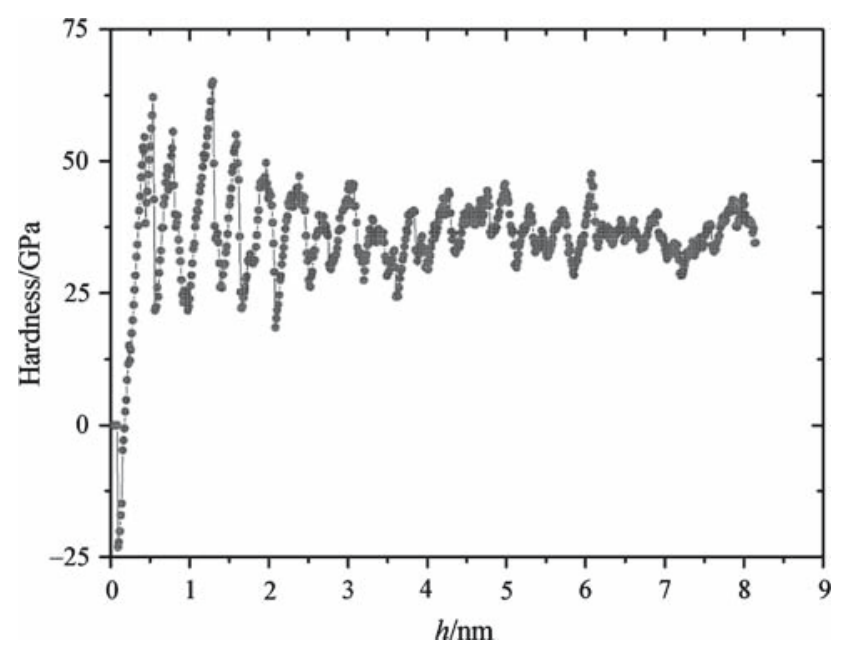

Fig. 9 The nanohardness versus the indentation depth curve obtained from MST/CST simulation [60]

Table 5 Comparison of MST and MD methods

\begin{tabular}{ll}
\hline MST & MD \\
\hline $\begin{array}{l}\text { Quasi-static } \\
\text { High efficiency: One tenth } \\
\text { of MD consumption }\end{array}$ & Short duration: $\leq 10^{-9} \mathrm{~s}$ \\
$\begin{array}{l}\text { MST/CST: Unified } \\
\text { potential }\end{array}$ & MD/FE: Mismatch of potential \\
& and constitutive relation \\
\hline
\end{tabular}

layer and the side surfaces of the sample are assumed to be fixed. In the MST/CST simulations, there are a MST region of $28.2 \mathrm{~nm} \times 28.2 \mathrm{~nm} \times 14.1 \mathrm{~nm}$ containing 936,975 atoms and 740 clusters with 405 nodes, containing 1,356,278 atoms. The loading step is $0.012 \mathrm{~nm}$ of indentation depth [60].

As an illustration, the simple $\mathrm{L}-\mathrm{J}$ potential $\varphi_{\mathrm{C}-\mathrm{Cu}}(r)=$ $\frac{C_{12}}{r^{12}}-\frac{C_{6}}{r^{6}}$ is adopted for both $\mathrm{Cu}-\mathrm{Cu}$ and $\mathrm{C}-\mathrm{Cu}$ atomic interactions, but $C_{12}=C r_{0}^{12}$ and $C_{6}=C r_{0}^{6}(C=1.9648 \mathrm{eV}$ and $r_{0}=0.23276 \mathrm{~nm}$ ) for $\mathrm{Cu}-\mathrm{Cu}$ interaction and $C_{12}=$ $2989.1 \mathrm{eV} \AA^{12}$ and $C_{6}=41.548 \mathrm{eV} \AA^{6}$ for $\mathrm{C}-\mathrm{Cu}$ atomic interaction [62].

For the simulations, the zero indentation depth is assumed to be the position where the interaction force between the tip and sample has just changed from attractive to repulsive. The hardness versus the indentation depth curves are shown in Fig. 9. It can be seen that there are three phases in the hardness variation.

Phase 1: The hardness starts from zero at zero indentation depth and increases with increasing indentation depth. In addition, the increase of hardness in this phase is in agreement with elastic contact theory [63].

Phase 2: At about $0.5 \mathrm{~nm}$ indentation depth (still under the indent of the spherical crown), plastic deformation appears and both load and hardness oscillate till the peak hardness at the indentation depth of $1.2 \mathrm{~nm}$, where the truncated cone has penetrates into the specimen.

Phase 3: Afterwards, the hardness decreases gradually till about $4 \mathrm{~nm}$ indentation depth (roughly 4 times the crown height) and dislocations seem to be saturated underneath the indenter. Beyond this indentation depth, the hardness nearly remains a constant with further indentation.

To summarize case study 4, the comparison of MD and MST/CST simulations are listed in Table 5.

Then, we use Table 6 to summarize the trans-scale features in all four case studies, to see if we could draw something in common for the possible paradigm in trans-scale mechanics.

From Table 6, one may notice that for all these transscale problems there are always some key trans-scale links, which come from the trans-scale coupling and govern the phenomena. So, a certain trans-scale paradigm in general needs further exploring.

\section{Possible modes of trans-scale mechanics}

According to the previous works outlined in introduction and the above-mentioned case studies, we intend to portrait some possible modes of trans-scale mechanics as a spring board to a more effective paradigm, even though future development in this direction must significantly change the picture.

\subsection{Possible mode 1: Non-equilibrium statistical mechanics}

Looking back to the development of statistical mechanics, it appears to be a trilogy. The first chapter of the trilogy is simple average. The equation of state of ideal gas is an excellent example.

$P V=R T=N_{0} k T$,

where $N_{0}$ is Avogadro's number. The implication of the equation is straightforward: the total thermal energy is the simple sum of the contribution from all degrees of atoms independently, namely $N_{0} k T$. The second chapter is the introduction of interaction between molecules. In this formulation, partition function plays a key role. Equilibrium thermodynamics and statistical physics are the sophisticated formulation of the paradigm. As a matter of fact, the principle of equal a priori probability at equilibrium state is the key in this formulation. This progress enables us to calculate all thermal properties of materials at equilibrium state. Physical mechanics proposed by Tsien [1] is a successful application of the paradigm to engineering.

Now, we may have to go further from physical mechanics to physical dynamics, as suggested by Kadanoff [64] to understand the non-equilibrium behaviors of media resulting from various kinetics at various levels. In these situations, the distribution function may no longer remain stationary, but 
Table 6 Summary of trans-scale features in case studies

\begin{tabular}{|c|c|c|c|}
\hline Case & Trans-scale formulation & Trans-scale link & Physical implication \\
\hline $\begin{array}{l}\text { 1. Nanometer shear band in } \\
\text { BMG's }\end{array}$ & At macroscopic level & $\delta_{\text {free volume }} \sim \sqrt{D \frac{\xi^{*}}{G^{*}}}$ & $\begin{array}{l}\text { Nano-pattern emerging due to } \\
\text { diffusion and creation of free } \\
\text { volume }\end{array}$ \\
\hline $\begin{array}{l}\text { 2. Spallation owing to microda- } \\
\text { mage evolution }\end{array}$ & $\begin{array}{l}\text { Coupled micro/ macroscopic } \\
\text { levels }\end{array}$ & $D e^{*}=\frac{a c^{*}}{L V^{*}}=\frac{t_{V}}{t_{i}} D^{*}=\frac{t_{V}}{t_{N}}=\frac{n_{N}^{*} c^{* 5}}{V^{*}}$ & Competition between macro- \\
\hline $\begin{array}{l}\text { 3. Interaction of probe tip and } \\
\text { liquid surface }\end{array}$ & $\begin{array}{l}\text { Coupled nano/micro/ } \\
\text { macroscopic levels }\end{array}$ & $y_{0 c} \sim \delta_{c} \sim\left(R \cdot \frac{A}{\gamma_{L V}}\right)^{1 / 3}$ & $\begin{array}{l}\text { Partition of nano/micro/ } \\
\text { macroscopic length scales }\end{array}$ \\
\hline $\begin{array}{l}\text { 4. MST/CST simulation of } \\
\text { nanoindentation }\end{array}$ & $\begin{array}{l}\text { Coupled nano/microscopic } \\
\text { levels and coupled } \\
\text { algorithm of atomic } \\
\text { oscillation/quasi-static } \\
\text { deformation }\end{array}$ & $\begin{array}{l}\text { Helmholtz free energy as a function } \\
\text { of the coordinates of atoms } A=A\left(\boldsymbol{x}_{i}\right) \text {, } \\
i=1, \ldots, N\end{array}$ & $\begin{array}{l}\text { Statistical thermodynamics and } \\
\text { lattice dynamics }\end{array}$ \\
\hline
\end{tabular}

Fig. 10 Illustration of distribution function at equilibrium and non-equilibrium states. a Stationary at equilibrium state; b Varying with time at nonequilibrium state
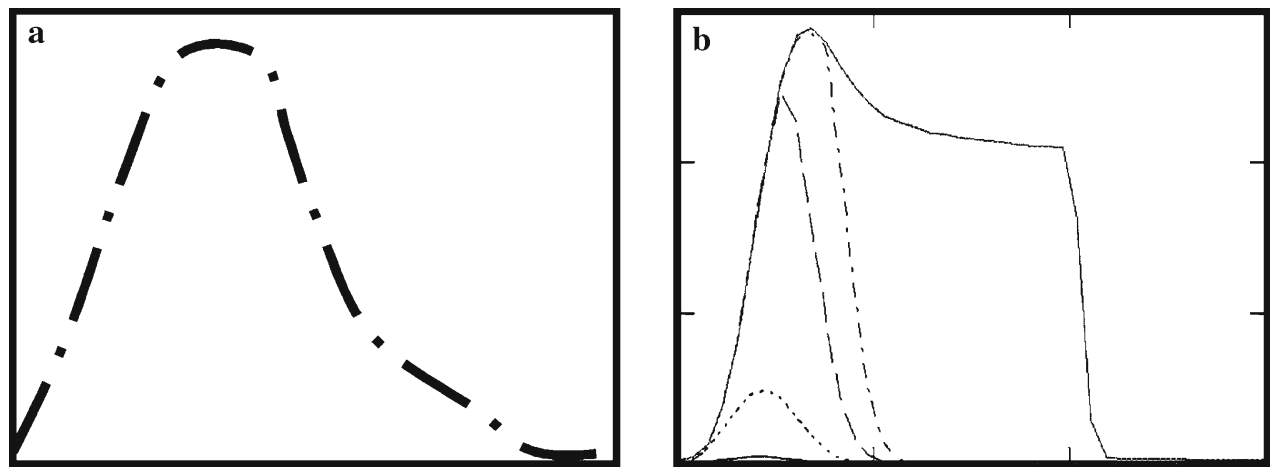

becomes unsteady (as case study 2 shows), see Fig. 10. In this mode, non-equilibrium statistical mechanics and thermodynamics will play a significant role to connect nano/microkinetics to macroscopic evolutionary behavior. In particular, for evolution-induced catastrophes, such as earthquake, avalanche etc., Kadanoff [64] proposed that we may have to understand the non-equilibrium behaviors of media in this manner.

\subsection{Possible mode 2: Coupled trans-scale equations and simultaneous solutions}

In 1992, in his closing lecture at 18th ICTAM, Barenblatt stated that in the mathematical models of such phenomena, the macroscopic equations of mechanics and the kinetic equations of the microstructural transformations form a unified set that should be solved simultaneously [65]. This approach can be sketched in Fig. 11. Obviously, this is a far-reaching idea. All case studies 1, 2 and 3 are on this track, but the trans-scale effects in case 1 are condensed to macroscopic level, but case 2 at macro/micro level and case 3 at macro/micro/nano level. However, generally speaking, to associate the macroscopic equations of continuum mechanics with the kinetic equations of microstructural transformations and to form a unified set is by no means an easy job, let alone to solve it simultaneously. The complexity shown in case study 2 might be the reflection of this issue. Hence, necessary evaluations and approximations must be introduced in the approach. In this aspect, Barenblatt emphasized the significance of Deborah number in these trans-scale problems. Perhaps, compared to the distinct spatial scale, the temporal scales may easily be ignored, though they crucially govern the association and competition of continuum mechanics and microstructural evolutions.

\subsection{Possible mode 3: Trans-scale algorithms based on atomic/molecular interaction}

With the rapid progress in computational technology, apart from the theoretical formulations of how the phenomena are physically trans-scale coupled, the trans-scale algorithms based on atomic interactions are also needed. As shown in case studies 3 and 4, there are huge gaps between length scales, greater than $10^{3}$ orders in one dimension from atomic to practical engineering objects, and between time scales, greater than $10^{6}$ orders from atomic oscillation to practical engineering processes, especially for mechanical processes. In this field, MD provided a very helpful guide but it is too much time-consuming, even for unrealistic fast processes 


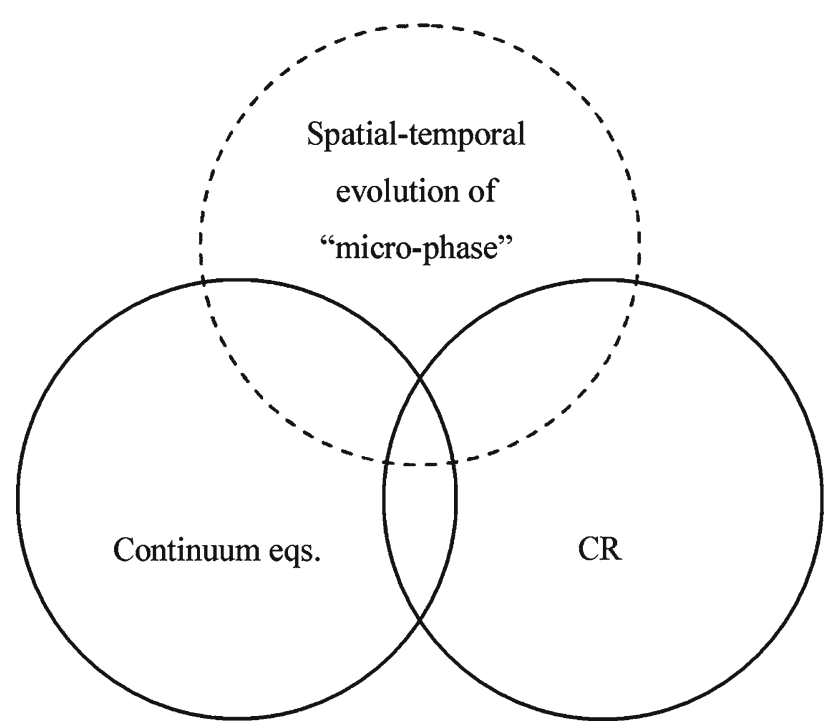

Fig. 11 An illustration of trans-scale coupling equations, as suggested by Barenblatt [65]

(higher than $10^{9} / \mathrm{s}$ ) in a small system (tens namometers). To accept this practical challenge, alternative trans-scale algorithms based on atomic/molecular interactions should be initiated. Perhaps, case study 4 is a representative example and both QC and MST/CST are encouraging steps in this direction. The crucial issues in this kind of algorithms might be how to introduce proper statistical treatments to bridge the huge gaps of spatial and temporal scales. Hence, novel and effective combinations of algorithms and statistics based on atomic/molecular interactions are anticipated.

In addition, it is worth noticing some further difficulties in engineering, which challenge trans-scale mechanics more severely. For instance, some macroscopic phenomena are governed by critical atomistic event, such as the sudden drops in stress-strain relations and rate-dependent behaviors owing to the appearance of dislocations and sliding at elevated temperature. In these cases, how to trace the minor but critical atomistic events and their evolutions among the enormous atoms may be a tricky and formidable job. The other example might be the prediction of catastrophic failure governed by non-linear trans-scale cascades. In this situation, the difficulties may come from the coupling of deterministic and stochastic processes, the coupling of heterogeneity and non-linear dynamics, as well as the coupling between atomic events and the variation of macroscopic variables.

In order to achieve relatively sophisticated or specific paradigms of trans-scale mechanics and to meet the challenging needs, to recall the statement on continuum mechanics made by Einstein should be very enlightening.

Einstein wrote [66]: continuum "avoids the consideration of a subdivision of matter down to 'real' material points, is the mechanics of so-called continuous media. This mechanics is characterized by the fiction that ... dependent in a continuous manner upon coordinates and time, and that the part of the interactions not explicitly given can be considered as surface forces (pressure forces) which again are continuous functions of location." Then, for further progress of mechanics, he suggested to coordinate atomistic entities to material points. "This occurred in the kinetic theory of gases and, in a general way, in statistical mechanics. ... These decisive progresses were paid for by the coordination of atomistic entities to the material points." However, compared to gases, the media with nano/micro-structures or emergent patterns are much more complicated and the coordination of atomistic entities to continuum is by no means a straightforward avenue. However, we should also be encouraged by the progresses made and the possible paradigms proposed in recent years. All these may imply that different from the direct coordination of atomistic entities to the material points in gases, the coordination of atomistic entities to the material points in the trans-scale mechanics would not be the oneto-one correspondence in appearance, but something similar in essential spirit. In this aspect, Chinese panorama philosophy, “神似" (likeness in spirit) not “形似” (likeness in form) may be a wise guide to the establishment of trans-scale mechanics.

Acknowledgment The long lasting cooperation with colleagues and graduates at LNM is greatly appreciated.

\section{References}

1. Tsien, S.H.: Physical Mechanics (in Chinese). Science Press, Beijing (1962)

2. Marder, M., Fineberg, J.: How things break. Phys. Today 499 , 24-29 (1996)

3. Conner, R.D., Johnson, W.L., Paton, N.E., Nix, W.D.: Shear bands and cracking of metallic glass plates in bending. J. Appl. Phys. 94, 904-911 (2003)

4. Shen, L.T., Zhao, S.D., Bai, Y.L., Luo, L.M.: Experimental study on the criteria and mechanism of spallation in an $\mathrm{Al}$ alloy. Int. J. Impact Eng. 12, 9-19 (1992)

5. Weinan, E., Engquist, B.: The heterogeneous multiscale methods. Commun. Math. Sci. 1, 87-132 (2003)

6. Galileo, G.: Dialogue Concerning Two New Sciences. William Andrew Publishing, New York (2001)

7. Schlichting, H.: Boundary Layer Theory. McGraw-Hill, New York (1968)

8. Hall, E.O.: The deformation and aging of mild steel. Proc. Phys. Soc. Lond. Sect. B 64, 747-753 (1951)

9. Petch, N.J.: The cleavage strength of polystrals. J. Iron Steel Inst. 173, 25-28 (1953)

10. Glimm, J., Sharp, D.H.: Multiscale science: A challenge for the twenty-first century. SIAM News 30, 1-7 (1997)

11. Sih, G.C.: Mesomechanics 2000 Role of Mechanics for Development of Science and Technology. In: Sih, G.C. (ed.) Tsinghua University Press, Beijing (2000)

12. Spaepen, F., Turnbull, D.: A mechanism for the flow and fracture of metallic glasses. Scripta Metall. 8, 563-568 (1974)

13. Spaepen, F.: A microscopic mechanism for steady state inhomogeneous flow in metallic glasses. Acta Metall. 25, 407-415 (1977) 
14. Falk, M.L., Shi, Y.: Strain localization in a molecular-dynamics model of a metallic glass. In: Egami, T., Greer, A.L., Inoue, A., Ranganathan, S. (eds.). Supercooled Liquids, Glass Transition, Bulk Metallic Glasses. Mat. Res. Soc. Proc. vol. 754, pp. 1-6. Pittsburgh (2003)

15. Wang, G.H., Pan, H., Ke, F.J., Xia, M.F., Bai, Y.L.: Study of mechanical properties of amorphous copper with molecular dynamics simulation. Chin. Phys. B 17, 1-5 (2008)

16. Steif, P.S., Spaepen, F., Hutchinson, J.W.: Strain localization in amorphous metals. Acta Metall. 30, 447-455 (1982)

17. Huang, R., Suo, Z.G., Prevost, J.H., Nix, W.D.: Inhomogeneous deformation in metallic glasses. J. Mech. Phys. Solids. 50, 10111027 (2002)

18. Dai, L.H., Yan, M., Liu, L.F., Bai, Y.L.: Adiabatic shear banding instability in bulk metallic glasses. Appl. Phys. Lett. 87, $141916(2005)$

19. Bruck, H.A., Rosakis, A.J., Johnson, W.L.: The dynamic compressive behavior of beryllium bearing bulk metallic glasses. J. Mater. Res. 11, 503-511 (1996)

20. Lewandowski, J.J., Greer, A.L.: Temperature rise at shear bands in metallic glasses. Nat. Mater. 5, 15-18 (2006)

21. Masumoto, T., Maddin, R.: Mechanical properties of palladium20 atomic \% silicon alloy quenched from the liqiud state. Acta Metall. 19, 725-741 (1971)

22. Donvan, P.E., Stobbs, W.M.: The structure of shear bands in metallic glass. Acta Metall. 29, 1419-1436 (1981)

23. Pekarskaya, E., Kim, C.P., Johnson, W.L.: In-situ transmission electron microscopy studies of shear bands in a bulk metallic glass based composite. J. Mater. Res. 16, 2513-2518 (2001)

24. Li, J., Wang, Z.L., Hufnagel, T.C.: Characterization of nanometerscale defects in metallic glasses by quantitative high-resolution transmission electron microscopy. Phys. Rev. B 65, 144201 (2002)

25. Liu, L.F.: Formation mechanism of shear band in bulk metallic glass (in Chinese). PhD Thesis, Institute of Mechanics, Chinese Academy of Sciences, Beijing (2006)

26. Dai, L.H., Bai, Y.L.: Basic mechanical behaviors and mechanics of shear bending in BMGs. Int. J. Impact Eng. (in press)

27. Conner, R.D., Dandliker, R.B., Scruggs, V., Johnson, W.L.: Dynamic deformation behavior of W-fiber/metallic-glass matrix composites. Int. J. Impact. Eng. 24, 435-444 (2000)

28. Wang, W.H., Dong, C., Shek, C.H.: Bulk metallic glasses. Mater. Sci. Eng. R 44, 45-89 (2004)

29. Liu, L.F., Dai, L.H., Bai, Y.L., Eckert, J.: Characterization of ratedependent shear behavior of Zr-based bulk metallic glass using shear-punch testing. J. Mater. Res. 21, 153-160 (2006)

30. Bai, Y.L., Ling, Z., Luo, L.M., Ke, F.J.: Initial development of microdamage under impact loading., ASME Trans. J. Appl. Mech. 59, 622-627 (1992)

31. Tuler, F.R., Butcher, B.M.: A criterion for the time dependence of dynamic fracture. Int. J. Fracture Mech. 4, 431-437 (1968)

32. Shen, L.T., Zhao, S.D., Bai, Y.L., Luo, L.M.: Experimental study on the criteria and mechanism of spallation in an Al alloy. Int. J. Impact Eng. 12, 9-19 (1992)

33. Meyers, M.A.: Dynamic Behaviour of Materials. Wiley, New York (1994)

34. Grady, D.E., Kipp, M.E. : Dynamic fracture and fragmentation. In: Asay, J.R., Shahinpoor, M. (eds.) High-Pressure Shock Compression of Solids, pp. 265-332. Springer, New York (1993)

35. Davison, L., Stevens, A.L.: Continuum measures of spall damage. J. Appl. Phys. 43, 988-994 (1972)

36. Han, W.S., Xia, M.F., Shen, L.T., Bai, Y.L.: Statistical formulation and experimental determination of growth rate of micrometre cracks under impact loading. Int. J. Solids Struct. 34, 2905-2925 (1997)

37. Bai, Y.L., Xia, M.F., Ke, F.J., Li, H.L.: Closed trans-scale statistical microdamage mechanics. Acta Mech. Sin. 18, 1-17 (2002)
38. Xia, M.F., Han, W.S., Ke, F.J., Bai, Y.L.: Statistical meso-scopic damage mechanics and damage evolution induced catastrophe (in Chinese). ADv. Mech. 25(1-40), 145-173 (1995)

39. Wang, H.Y., Bai, Y.L., Xia, M.F., Ke, F.J.: Spallation analysis with a closed trans-scale formulation of damage evolution. Acta Mech. Sin. 20, 400-407 (2004)

40. Wang, H.Y., Bai, Y.L., Xia, M.F., Ke, F.J.: Microdamage evolution, energy dissipation and its trans-scale effects on macroscopic failure. Mech. Mater. 38, 57-67 (2006)

41. Bai, Y.L., Han, W.S., Bai, J.: A statistical evolution equation of microdamage and its application. ASTM STP 1315, 150-162 (1997)

42. Bai, Y.L., Wang, H.Y.: The significance of the intrinsic Deborah numbers in failure. In: Hong, Y.S. (ed.) Advances in Applied Mechanics. Science Press, Beijing (2004)

43. Freund, J., Halbritter, J., Horber, J.K.H.: How dry are dried samples? Water adsorption measured by STM. Microsc. Res. Tech. 44, 327-338 (1999)

44. Liu, N., Bai, Y.L., Xia, M.F., Ke, F.J.: Combined effect of surface tension, gravity and van der Waals force induced by a non-contact probe tip on the shape of liquid surface. Chin. Phys. Lett. 22, 20122015 (2005)

45. Israelachvili, J.N.: Intermolecular and Surface Forces. Academic Press, San Diego (1985)

46. Cortat, F.P.A., Miklavcic, S.J.: Using stable and unstable profiles to deduce deformation limits of the air-water interfaces. Langmuir 20, 3208-3220 (2004)

47. Wang, H.Y., Hu, M., Liu, N., Xia, M.F., Ke, F.J., Bai, Y.L.: Multiscale analysis of AFM tip and surface interactions. Chem. Eng. Sci. 62, 3589-3594 (2007)

48. Liu, N.: The distortion and instability of liquid surface induced by a sub-microscale probe. [Master's Thesis], Institute of Mechanics, Chinese Academy of Sciences, Beijing (2005) (in Chinese)

49. Haile, J.M.: Molecular Dynamics Simulation. Wiley, New York (1997)

50. Oliver, W.C., Pharr, G.M.: An improve technique for determining hardness and elastic-modulus using load and displacement sensing indentation experiments. J. Mater. Res. 7, 1564-1583 (1992)

51. Ma, Q., Clark, D.R.: Size-dependent hardness of silver single crystals. J. Mater. Res. 10, 853-863 (1995)

52. McElhaney, K.W., Vlassak, J.J., Nix, W.D.: Determination of indenter tip geometry and indentation contact area for depthsensing indentation experiments. J. Mater. Res. 13, 1300-1306 (1998)

53. Liu, Y., Ngan, A.H.W.: Depth dependence of hardness in copper single crystals measured by nanoindentation. Script Mater. 44, 237-241 (2001)

54. Wei, Y.G., Wang, X.Z., Wu, X.L., Bai, Y.L.: Theoretical and experimental researches of size effect in micro-indentation test. Sci. China, A 44, 74-82 (2001)

55. Chen, S.D., Ke, F.J.: MD simulation of the effect of contact area and tip radius on nanoindentation. Sci. China Ser. G-Phys. Astron. 47, 101-112 (2004)

56. Noreyan, A., Amar, J.G., Marinescu, I.: Molecular dynamics simulations of nanoindentation beta-SiC with diamond indenter. Mater. Sci. Eng. B Solid State Mater. Adv. Technol. 117, 235-240 (2005)

57. Dupuy, L.M., Tadmor, E.B., Miller, R.E., Phillips, R.: Finitetemperature quasicontinuum: Molecular dynamics without all the atoms. Phys. Rev. Lett. 95, 060202 (2005)

58. Hu, M., Wang, H.Y., Bai, Y.L., Xia, M.F., Ke, F.J.: Cluster statistical thermodynamics - to efficiently calculate quasi-static deformation at finite temperature based on molecular potential. In: IUTAM Symposium on Mechanical Behavior and MicroMechanics of Nanostructured Materials, pp. 163-170, Beijing, China, June 27-30, 2005 (2007) 
59. Wang, H.Y., Hu, M., Xia, M.F., Ke, F.J., Bai, Y.L.: Molecular/ cluster statistical thermodynamics methods to simulate quasistatic deformations at finite temperature. Int. J. Solids Struct. (in press)

60. Hu, M.: Statistical quasicontinuum method at nano/micro-meter scales and its applications (in Chinese). Ph.D. Thesis, Institute of Mechanics, Chinese Academy of Sciences, Beijing (2006)

61. Born, M., Huang, K.: Dynamical Theory of Crystal Lattices. Oxford Press, Landon (1954)

62. Ellis, D.E., Mundim, K.C., Fuks, D., Dorfman, S., Berner, A.: Modeling of copper-carbon solid solutions. Mater. Sci. Semiconductor Process. 3, 123-127 (2000)
63. Sneddon, I.N.: The relation between load and penetration in the axisymmetric boussinesq problem for a punch of arbitrary profile. Int. J. Eng. Sci. 3, 47-57 (1965)

64. Kadanoff, L.P.: Statistical Physics (Statics, Dynamics and Renormalization). World Scientific, Singapore (2000)

65. Barenblatt, G.I.: Micromechanics of fracture, Theoretical and Applied Mechanics. Bodner S.R., Singer J., Solan A., Hashin J. (eds.) Elesevier, Amsterdam, pp. 25-52 (1992)

66. Einstein, A.: Physics and Reality, in Einstein, Ideas and Opinions. Crown, New York (1954) 